\title{
Utility of Synthetic, Medicinal and Pharmaceutical Chemistry in Drug Design
}

\section{Prakash Prajapat}

Department of Chemistry, Mehsana Urban Institute of Sciences, Ganpat University, Mehsana, Gujarat, India

*Corresponding author: Prakash Prajapat, Department of Chemistry, Mehsana Urban Institute of Sciences, Ganpat University, Mehsana-384 012, Gujarat, India, Tel: 07891554090; E-mail: prajapatprakash11@yahoo.in

Rec date: March 13, 2018; Acc date: March 21, 2018; Pub date: March 26, 2018

Copyright: () 2018 Prajapat P. This is an open-access article distributed under the terms of the Creative Commons Attribution License, which permits unrestricted use, distribution, and reproduction in any medium, provided the original author and source are credited.

\begin{abstract}
Drug design is a creative act of the same magnitude as composing, sculpting, or writing. The results can touch the lives of millions and bring dollars of millions. Drug design, sometimes referred to as rational drug design or simply rational design, is the inventive process of finding new medications based on the knowledge of a biological target. Similarly, drug development is the process of bringing a new pharmaceutical drug to the market once a lead compound has been identified through the process of drug discovery. It includes pre-clinical research (microorganisms/animals) and clinical trials (on humans) and may include the step of obtaining regulatory approval to market the drug. This review helps in the development of newer molecules for future drug discovery.
\end{abstract}

Keywords: Drug design; Pharmaceutical drug; Pre-clinical research; Drug discovery

\section{Introduction}

The development of new active therapeutic agents is currently a critical and challenging issue to the pharmaceutical industry. Medicinal and pharmaceutical chemistry is a scientific discipline at the interaction of chemistry and pharmacology involved with designing, synthesizing and developing pharmaceutical drugs. The process of drug discovery involves the identification of candidates, synthesis, characterization, screening, and assays for therapeutic efficacy. Once a compound has shown its value in these tests, it will begin the process of drug development prior to clinical trials [1-7]. Pharmaceutical chemistry is focused on quality aspects of medicines and aims to assure fitness for the purpose of medicinal products. The science of medicinal chemistry involves the design and synthesis of novel drugs based on an understanding of how drugs work in the body at the molecular level. Medicinal Chemistry is a multifaceted discipline that encompasses synthetic organic chemistry, natural products chemistry, enzymology, chemical biology, structural biology and computational methods, all of which is aimed at the discovery and development of new therapeutic agents. Scientists in the field are well positioned to work as part of an interdisciplinary team that uses chemical structural principles to design effective drugs and diagnostic agents $[8,9]$. The techniques are used in the fields of computational chemistry, drug design, computational biology and materials science for studying molecular systems ranging from small chemical systems to large biological molecules and material assemblies. Nowadays, drug design and development are followed by QSAR analysis, optimizing pharmacokinetic and pharmacodynamic properties. Applications involving genomics, proteomics, cheminformatics, bioinformatics, high-throughput X-ray crystallography, targeted combinatorial libraries, etc. mostly considered to raise the output in the form of quality leads. Many works are still needed to minimize the time, expenditure, and attrition rate in the drug discovery process simultaneously addressing the huge unmet medical need across the world [10,11] (Figure 1). Furthermore, Computer Aided Drug Designing (CADD) techniques to assist organic and medicinal chemists in drug design, development, and hit-optimization steps during the drug discovery process.

A majority of working medicinal chemists have degrees in organic chemistry, rather than medicinal chemistry. The chief role of medicinal chemist is to design and synthesized the target structures required. Therefore, the medicinal chemist is an essential member, who identify whether proposed target structures are likely to be stable and whether they can be synthesized or not. Chemists and medicinal chemists make important contribution at various stages of drug research programme. The medicinal chemist is most involved in drug discovery and drug design. Organic synthetic chemists are involved in synthesizing drugs by various synthetic techniques, while analytical chemists are involved in quality control, data preparation and purity specifications. Other practical skills acquired by different chemistry disciplines are extraction of plant synthetic products, chromatography, distillation, crystallization which have a vital role to designate a lead compound in drug research programme. Multiple skills are required in order to synthesize analogues of lead compounds to carry out structure activity relationship. Spectroscopy experts have an important role in interpretation of various spectral studies in determining structure of a synthetic analogue. Development chemists generally plan and devise a large scale (bulk) synthesis. A wide range of synthetic and medicinal utility shown by heterocyclic hybrids inspired organic and medicinal chemists to pursue the synthesis of newer motifs and evaluate their pharmacophoric importance [12-17]. Finally, the varieties of chemistry have contributed to life processes and to the efforts to advance the quality of life as well as to the development of society from synthetic, medicinal, pharmaceutical point of view. 


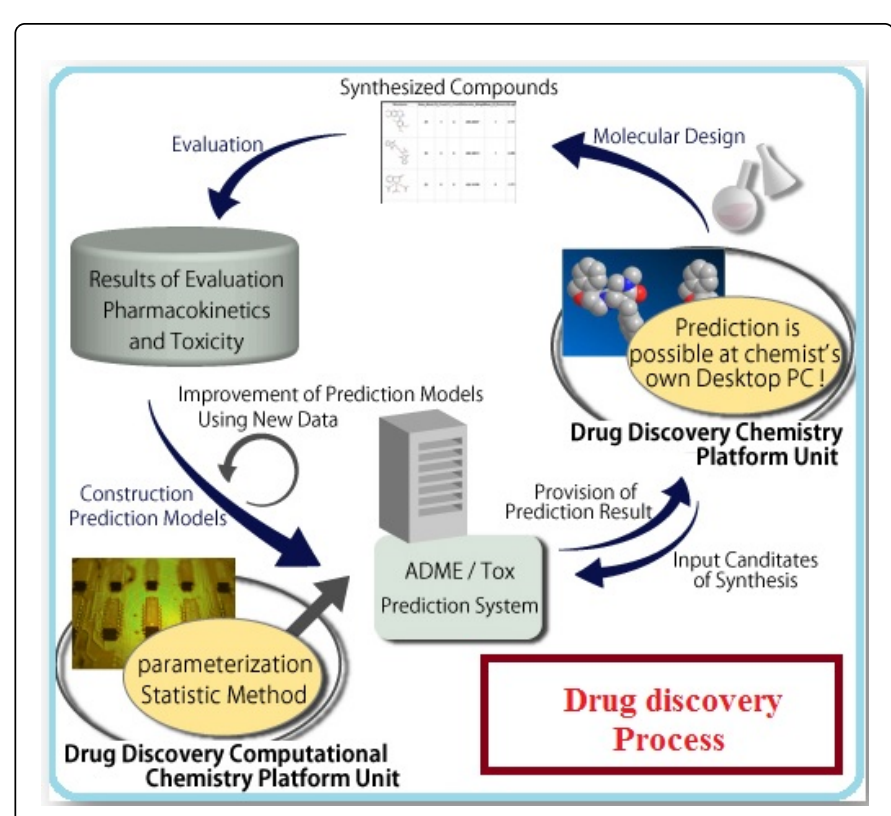

Figure 1: Different steps of Drug Discovery Process.

\section{Conclusion}

Finally, this opinion helps to find potential future directions on the drug design and development of new effective and specific analogues in synthetic, medicinal and pharmaceutical chemistry. Hence, this information would give rise to design of better molecules with enhanced drug properties and higher specificity, and together with development of novel synthetic strategies.

\section{Acknowledgement}

I thankful to chemistry staff members of the Mehesana Urban Institute of Sciences, Ganpat University, Mehsana, Gujarat for their support.

\section{References}

1. Prajapat P, Vaghani H, Agarwal S, Talesara GL (2017) Synthetic and Medicinal Chemistry in Drug Discovery: Needs for Today. Ann Med Chem Res 3: 1021.

2. Prajapat P, Agarwal S, Talesara GL (2017) Significance of Computer Aided Drug Design and 3D QSAR in Modern Drug Discovery. J Med Org Chem 1: 1.

3. Prajapat P (2017) Utility of Drug Discovery in Medicinal and Organic Chemistry. Mod Chem Appl 5: e123.
4. Prajapat P (2018) Importance of Benzothiazole Motif in Modern Drug Discovery: Introduction. Mod Appro Drug Des 1: MADD.000517.

5. Prajapat P (2018) Some Important Applications of Recent Advances in Applied Chemical Science. Mod Chem Appl 6: e124.

6. Prajapat P (2018) Role of Combinatorial, Medicinal \& Biological chemistry in drug discovery development: An Introduction. Arc Org Inorg Chem Sci 1: AOICS.MS.ID.000104.

7. Agarwal S, Kalal P, Gandhi D, Prajapat P (2017) Thiazole containing Heterocycles with CNS activity. Curr Drug Discov Technol 14.

8. Prajapat P, Yogi P, Talesara GL (2015) Synthesis of Biological Significant New 1-(1,3-benzoxazol-2-yl)guanidine Derivatives. Journal of Chemistry and Chemical Sciences 5: 670-681.

9. Prajapat P, Talesara GL (2016) Synthesis and Anti-inflammatory Screening of Some Mono and Bis-Alkoxyphthalimide Linked Benzimidazole and their Quinazoline and Pyrimidine Derivatives. J Heterocyclic Chem 53: 163-1610.

10. Prajapat $P$ (2018). Role of organic, medicinal \& pharmaceutical chemistry in drug design: introduction. J Nanomed Res 7: 69-70.

11. Prajapat P, Kumawat M, Talesara GL, Kalal P, Agarwal S, et al. (2018) Benzimidazole scaffold as a versatile biophore in drug discovery: A review. Chemistry \& Biology Interface 8: 1-10.

12. Prajapat P, Kumawat M, Kherodiya B, Talesara GL (2016) An expedient synthesis and antimicrobial evaluation of ethoxyphthalimido derivatives of pyrimido[4,5-e]pyrimidine analogues from 1-(1H-benzimidazol-2yl)guanidine. J Indian Chem Soc 93: 539-544.

13. Kumawat M, Kherodiya B, Prajapat P, Talesara GL (2015) Synthesis of alkoxyphthalimide derivatized oxoimidazolidinyl oxazolo/thiazolo dihydropyrimidine and oxoimidazolidinyl tetrahydropyrimidine via common Schiff base intermediate and evaluation of their antibacterial activity. Indian J Chem 54B: 117-127.

14. Prajapat P, Rathore KK, Hussain N, Yogi PP, Talesara GL (2015) Synthesis of novel pyrimidines, pyrimidopyrimidines and their oxygen substituted hydroxylamine derivatives as potential pharmacological interest. Iranian Journal of Organic Chemistry 7: 1605-1612.

15. Kherodiya B, Prajapat P, Kumawat M, Talesara GL (2015) Synthesis and antimicrobial evaluation of bis imidazolidinone assembled dihydropyridine ethoxyphthalimide derivatives. Iranian Journal of Organic Chemistry 7: 1661-1668.

16. Prajapat P, Rathore, KK, Gandhi D, Agarwal S, Hussain N, et al. (2016) A Facile Synthesis of Biologically Significant 2-(1,3-benzothiazol-2ylimino)-1,3-thiazolidin-4-one / 3-(1,3-benzothiazol-2-yl)-2thioxoimidazolidin-4-on Analogues from 1-(1,3-benzothiazol-2yl)thiourea and their Alphahydroxylamine Derivatives. Iranian Journal of Organic Chemistry 8: 1795-1801.

17. Hussain N, Ahmed M, Khan S, Joshi A, Yogi P, et al. (2017) One-pot Synthesis of Bis[1-N,7-N-diethoxyphthalimido-4,4'-\{3,5-dimethyl-4-(4N,N-dimethyl Aminophenyl)-4,7-dihydro-1H-dipyrazolo[3,4-b;4,3'e]Pyridin-8-yl\}-phenyl] Compounds and Their Antiviral Evaluation Against Cytomegalovirus (CMV) and Varicella-zoster Virus (VZV) in Human Embryonic Lung (HEL) Cells. J Heterocyclic Chem 54: 993-998. 